\title{
A Deeper Look at the New Milky Way Satellites: Sagittarius II, Reticulum II, Phoenix II, and Tucana III*
}

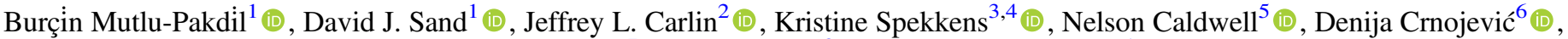 \\ Allison K. Hughes ${ }^{7}$, Beth Willman ${ }^{8}$ (iD, and Dennis Zaritsky ${ }^{1}$ (iD) \\ ${ }^{1}$ Department of Astronomy/Steward Observatory, 933 North Cherry Avenue, Rm. N204, Tucson, AZ 85721-0065, USA; bmutlupakdil@as.arizona.edu \\ ${ }^{2}$ LSST, 950 North Cherry Avenue, Tucson, AZ 85719, USA \\ ${ }^{3}$ Department of Physics, Engineering Physics and Astronomy, Queen's University, Kingston, Ontario, Canada \\ ${ }^{4}$ Department of Physics, Royal Military College of Canada, P.O. Box 17000, Station Forces, Kingston, ON K7L 7B4, Canada \\ ${ }^{5}$ Harvard-Smithsonian Center for Astrophysics, 60 Garden Street, Cambridge, MA 02138, USA \\ 6 Texas Tech University, Physics \& Astronomy Department, Box 41051, Lubbock, TX 79409-1051, USA \\ ${ }^{7}$ University of Arizona, Department of Physics, 1118 E. Fourth Street, Tucson, AZ 85721, USA \\ ${ }^{8}$ LSST and Steward Observatory, 933 North Cherry Avenue, Tucson, AZ 85721, USA \\ Received 2018 April 20; revised 2018 June 12; accepted 2018 June 13; published 2018 August 7
}

\begin{abstract}
We present deep Magellan/Megacam stellar photometry of four recently discovered faint Milky Way satellites: Sagittarius II (Sgr II), Reticulum II (Ret II), Phoenix II (Phe II), and Tucana III (Tuc III). Our photometry reaches 2-3 magnitudes deeper than the discovery data, allowing us to revisit the properties of these new objects (e.g., distance, structural properties, luminosity measurements, and signs of tidal disturbance). The satellite colormagnitude diagrams show that they are all old $(\sim 13.5 \mathrm{Gyr})$ and metal poor $([\mathrm{Fe} / \mathrm{H}] \lesssim-2.2)$. Sgr II is particularly interesting, as it sits in an intermediate position between the loci of dwarf galaxies and globular clusters in the sizeluminosity plane. The ensemble of its structural parameters is more consistent with a globular cluster classification, indicating that Sgr II is the most extended globular cluster in its luminosity range. The other three satellites land directly on the locus defined by Milky Way ultra-faint dwarf galaxies of similar luminosity. Ret II is the most elongated nearby dwarf galaxy currently known for its luminosity range. Our structural parameters for Phe II and Tuc III suggest that they are both dwarf galaxies. Tuc III is known to be associated with a stellar stream, which is clearly visible in our matched-filter stellar density map. The other satellites do not show any clear evidence of tidal stripping in the form of extensions or distortions. Finally, we also use archival H I data to place limits on the gas content of each object.
\end{abstract}

Key words: galaxies: dwarf - galaxies: individual (Sagittarius II, Reticulum II, Phoenix II, Tucana II) - galaxies: structure

Supporting material: machine-readable tables

\section{Introduction}

Ultra-faint dwarf galaxies are the smallest, most dark matterdominated, and least chemically enriched stellar systems in the universe. They are important targets for understanding the physics of dark matter and galaxy formation on the smallest scales. The details of their nature provide crucial empirical input for verifying formation scenarios of the Milky Way (MW).

Many new MW satellites have been discovered in the last few years (e.g., Balbinot et al. 2013; Belokurov et al. 2014; Laevens et al. 2014, 2015; Bechtol et al. 2015; DrlicaWagner et al. 2015, 2016; Kim et al. 2015a; Koposov et al. 2015a, 2017, 2018; Martin et al. 2015; Torrealba et al. 2016, 2018), but some of these new discoveries have been called into question by recent deep-imaging campaigns (Conn et al. 2018a, 2018b), and most of these new objects are poorly constrained in terms of their stellar population, structural parameters, distance, and luminosity. This paper aims to derive more accurate constraints on four ultra-faint satellites-Sagittarius II (Sgr II), Reticulum II (Ret II), Phoenix II (Phe II), and Tucana III (Tuc III)—by analyzing deep photometric observations.

\footnotetext{
* This paper includes data gathered with the $6.5 \mathrm{~m}$ Magellan Telescopes at Las Campanas Observatory, Chile.
}

Sgr II was discovered in the Pan-STARRS (PS1) survey by Laevens et al. (2015). It is especially interesting, as it is either the most compact dwarf galaxy or the most extended globular cluster in its luminosity-size range. Ret II and Phe II were discovered in the first-year Dark Energy Survey (DES) independently by Bechtol et al. (2015) and Koposov et al. (2015a). As with several recently discovered satellites in DES, the derived structural parameters differ between these studies. Deeper photometry is necessary to resolve this discrepancy. Tuc III was discovered in the second-year DES data by DrlicaWagner et al. (2015). Spectroscopic observations have been unable to conclusively determine its dynamical status and dark matter content (Simon et al. 2017), and it has a stellar stream extending at least $\pm 2^{\circ}$ from its core (Drlica-Wagner et al. 2015; Shipp et al. 2018), which strongly influences the measurements of its size and shape from the discovery DECam data.

Dark matter may annihilate to produce gamma-rays (e.g., Gunn et al. 1978; Bergström \& Snellman 1988; Baltz et al. 2008). The J-factor is a measure of the strength of this predicted signal and can be estimated using stellar kinematics. Due to their large dark matter content, relative proximity, and low astrophysical foregrounds, the MW dwarf galaxies are promising targets for indirect dark matter searches. However, it can be hard to obtain reliable J-factor estimates due to the difficulty of obtaining spectroscopy on these faint objects. 
Recently, Pace \& Strigari (2018) derived a scaling relation to estimate the J-factor based on the observable properties, such as half-light radius, without the full dynamical analysis. This makes deeper photometric data particularly important to precisely measure the structural parameters for these galaxies. We note that spectroscopic observations have only been published for Ret II and Tuc III, not Sgr II, and Phe II. Spectroscopic data of Tuc III only provides an upper limit on its J-factor (Simon et al. 2017). A tentative and controversial gamma-ray detection from Ret II (Geringer-Sameth et al. 2015; Hooper \& Linden 2015) demands detailed measurement of all of its physical parameters, and an evaluation of its dynamical status.

It is also possible to search for signs of tidal disruption via deep wide-field imaging (e.g., Coleman et al. 2007b; Sand et al. 2009; Muñoz et al. 2010; Sand et al. 2012; Roderick et al. 2015; Carlin et al. 2017), and this can be an important probe of the dynamical status of a satellite. Follow-up observations of these signs of structural disturbance have led to additional evidence of disruption in the form of extended distributions of RR Lyrae stars (e.g., Garling et al. 2018) and velocity gradients (Adén et al. 2009; Collins et al. 2017) in some new MW satellites.

This paper is organized as follows. In Section 2 we describe the observations, photometry, and calibration of our data. Section 3 details our photometric analysis, including new distance measurements, structural parameter measurements, and a search for signs of extended/disturbed structure. We also use archival $\mathrm{H} \mathrm{I}$ data to place upper limits on the gas content of each object. In Section 4 we discuss the individual objects in turn. Finally, we summarize and conclude in Section 5.

\section{Observations and Data Reduction}

We observed our targets with Megacam (McLeod et al. 2006) at the $f / 5$ focus at the Magellan Clay telescope in the $g$ and $r$ bands. The data were mostly taken during a single run on 2015 October 12-13, and was supplemented by some observations taken on 2017 October 17. Data from this program on Eridanus II was previously presented in Crnojević et al. (2016), although we will not discuss those observations further here. Magellan/Megacam has 36 CCDs, each with $2048 \times 2048$ pixels at 0 " $08 /$ pixel (which were binned $2 \times 2$ ), for a total field of view $(\mathrm{FoV})$ of $24^{\prime} \times 24^{\prime}$. We obtained $300 \mathrm{~s}$ individual exposures in $g$ and $r$ bands, and small dithers were used to cover the chip gaps in the final stacks. We reduced the data using the Megacam pipeline developed at the HarvardSmithsonian Center for Astrophysics by M. Conroy, J. Roll, and B. McLeod, including detrending the data, performing astrometry, and stacking the individual dithered frames. Astrometric solutions for each science exposure were derived using Two Micron All-Sky Survey catalog (2MASS; Skrutskie et al. 2006). Typical residuals for the matches to the 2MASS catalog are approximately 120 mas (McLeod et al. 2015).

We perform point-spread function (PSF) fitting photometry on the final image stacks, using the DAOPHOTII/ALLSTAR package (Stetson 1994) and following the same methodology described in Sand et al. (2009). In short, we use a quadratically varying PSF across the field to determine our PSF model. We run ALLSTAR twice: first on the final stacked image, and then on the first round's star-subtracted image, in order to recover fainter sources. We remove objects that are not point sources by culling our ALLSTAR catalogs of outliers in $\chi$ versus magnitude, magnitude error versus magnitude, and sharpness versus magnitude space. We positionally match our source catalogs derived from $g$ and $r$ filters with a maximum match radius of 0.15 , and create our final catalogs by only keeping those point sources detected in both bands.

We calibrate the photometry for Sgr II by matching to the PS1 survey (Chambers et al. 2016; Flewelling et al. 2016; Magnier et al. 2016). The Ret II, Phe II, and Tuc III fields were calibrated by matching to the DES DR1 catalog (DES Collaboration 2018). We note that the DES did not cover the Sgr II field, and the PS1 did not cover the others. A zeropoint and color term were fit for all fields and filters. We use all stars within the FoV with $17.5<g<20.5$ and $17.5<r<20.5$. A final overall scatter about the best-fit zeropoint is $\lesssim 0.01 \mathrm{mag}$ in all of our photometric bands. We correct for MW extinction on a star-by-star basis using the Schlegel et al. (1998) reddening maps with the coefficients from Schlafly \& Finkbeiner (2011), which were evaluated according to an Fitzpatrick (1999) reddening law with normalization $N=0.78$. Specifically, we adopted the coefficients of 3.172 and 2.271 for PS1 $g$ and $r$; and 3.237 and 2.176 for DES $g$ and $r$ to use with $E(B-V)$. The extinction-corrected photometry is used throughout this work.

To determine our photometric errors and completeness as a function of magnitude and color, we perform a series of artificial star tests with the DAOPHOT routine ADDSTAR. Similar to Sand et al. (2012), we place artificial stars into our images on a regular grid (10-20 times the image FWHM). We assign the $r$ magnitude of the artificial stars randomly from 18 to 29 mag with an exponentially increasing probability toward fainter magnitudes, and the $g$ magnitude is then randomly selected based on the $g-r$ color over the range $-0.5-1.5$ mag, with uniform probability. Ten iterations are performed on each field for a total of $\sim 100,000$ artificial stars each. These images are then photometered in the same way as the unaltered image stacks and the same stellar selection criteria on $\chi$, magnitude, magnitude error, and sharpness were all applied to the artificial star catalogs to determine our completeness and average magnitude uncertainties.

We present an observation log and our completeness limits in Table 1. Our photometry reaches $\sim 2-3$ mag deeper than the original discovery data for each object. Tables 2-5 present our full Sgr II, Ret II, Phe II, and Tuc III catalogs, which include the calibrated magnitudes (uncorrected for extinction) along with their DAOPHOT uncertainty, as well as the Galactic extinction values derived for each star (Schlafly \& Finkbeiner 2011).

\section{Analysis \\ 3.1. Color-Magnitude Diagrams}

Figure 1 (Sgr II) and 2 (Ret II, Phe II, and Tuc III) show the final color-magnitude diagrams (CMDs), which include stars within one half-light radius of the center (see Section 3.3). Magenta error bars show the mean photometric errors determined from artificial stars. The error bars are plotted at an arbitrary color for convenience. Blue open diamonds are the blue horizontal-branch star (HB) candidates in our FoV, which are selected within a filter with a color of $<0.2$ and a span of $\pm 0.5 \mathrm{mag}$, centered on the $\mathrm{HB}$ sequence of a metal-poor PARSEC isochrone (Bressan et al. 2012). Right panels show background-subtracted binned Hess diagrams, which encode 
Table 1

Summary of Observations and Field Completeness

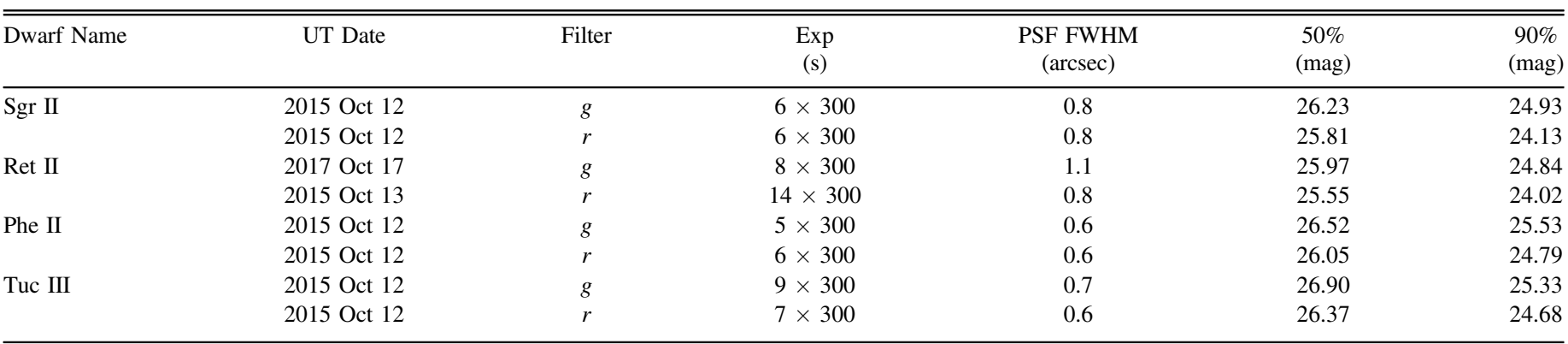

Table 2

Sgr II Photometry in the PS1 Photometric System

\begin{tabular}{|c|c|c|c|c|c|c|c|c|}
\hline Star No. & $\begin{array}{c}\alpha \\
(\operatorname{deg} \mathbf{J} 2000.0)\end{array}$ & $\begin{array}{c}\delta \\
(\operatorname{deg} J 2000.0)\end{array}$ & $\begin{array}{c}g \\
(\mathrm{mag})\end{array}$ & $\begin{array}{c}\delta g \\
(\mathrm{mag})\end{array}$ & $\begin{array}{c}A_{g} \\
(\mathrm{mag})\end{array}$ & $\begin{array}{c}r \\
(\mathrm{mag})\end{array}$ & $\begin{array}{c}\delta r \\
(\mathrm{mag})\end{array}$ & $\begin{array}{c}A_{r} \\
(\mathrm{mag})\end{array}$ \\
\hline 0 & 297.93544 & -22.131170 & 18.87 & 0.01 & 0.35 & 17.88 & 0.01 & 0.25 \\
\hline 1 & 297.93544 & -22.219629 & 18.31 & 0.01 & 0.35 & 17.80 & 0.01 & 0.25 \\
\hline 3 & 297.93562 & -22.140471 & 19.61 & 0.01 & 0.35 & 19.12 & 0.01 & 0.25 \\
\hline 4 & 297.93651 & -22.144029 & 19.69 & 0.01 & 0.35 & 19.15 & 0.01 & 0.25 \\
\hline
\end{tabular}

(This table is available in its entirety in machine-readable form.)

Table 3

Ret II Photometry in the DES Photometric System

\begin{tabular}{|c|c|c|c|c|c|c|c|c|}
\hline Star No. & $\begin{array}{c}\alpha \\
(\operatorname{deg} \mathrm{J} 2000.0)\end{array}$ & $\begin{array}{c}\delta \\
(\operatorname{deg} \mathrm{J} 2000.0)\end{array}$ & $\begin{array}{c}g \\
(\mathrm{mag})\end{array}$ & $\begin{array}{c}\delta g \\
(\mathrm{mag})\end{array}$ & $\begin{array}{c}A_{g} \\
(\mathrm{mag})\end{array}$ & $\begin{array}{c}r \\
(\mathrm{mag})\end{array}$ & $\begin{array}{c}\delta r \\
(\mathrm{mag})\end{array}$ & $\begin{array}{c}A_{r} \\
(\mathrm{mag})\end{array}$ \\
\hline 0 & 53.565994 & -54.247698 & 18.04 & 0.01 & 0.06 & 17.03 & 0.01 & 0.04 \\
\hline 1 & 53.566061 & -54.247761 & 18.26 & 0.02 & 0.06 & 17.01 & 0.01 & 0.04 \\
\hline 3 & 53.576270 & -54.168395 & 18.32 & 0.01 & 0.05 & 17.45 & 0.01 & 0.03 \\
\hline 4 & 53.578226 & -53.952364 & 18.11 & 0.01 & 0.05 & 15.54 & 0.01 & 0.03 \\
\hline
\end{tabular}

(This table is available in its entirety in machine-readable form.)

Table 4

Phe II Photometry in the DES Photometric System

\begin{tabular}{lcccccccc}
\hline \hline Star No. & $\begin{array}{c}\alpha \\
(\text { deg J2000.0) }\end{array}$ & $\begin{array}{c}\delta \\
(\text { deg J2000.0) }\end{array}$ & $\begin{array}{c}g \\
(\mathrm{mag})\end{array}$ & $\begin{array}{c}\delta g \\
(\mathrm{mag})\end{array}$ & $\begin{array}{c}A_{g} \\
(\mathrm{mag})\end{array}$ & $\begin{array}{c}r \\
(\mathrm{mag})\end{array}$ & $\begin{array}{c}\delta r \\
(\mathrm{mag})\end{array}$ & $\begin{array}{c}A_{r} \\
(\mathrm{mag})\end{array}$ \\
\hline 0 & 354.65054 & -54.405307 & 19.50 & 0.01 & 0.04 & 18.05 & 0.01 \\
1 & 354.65970 & -54.537558 & 19.93 & 0.01 & 0.04 & 19.00 & 0.01 \\
2 & 354.66431 & -54.542757 & 18.77 & 0.01 & 0.04 & 17.74 & 0.03 \\
3 & 354.67117 & -54.417531 & 18.26 & 0.01 & 0.04 & 17.72 & 0.01 \\
4 & 354.68689 & -54.386980 & 19.83 & 0.02 & 0.04 & 0.03 \\
\hline
\end{tabular}

(This table is available in its entirety in machine-readable form.)

Table 5

Tuc III Photometry in the DES Photometric System

\begin{tabular}{|c|c|c|c|c|c|c|c|c|}
\hline Star No. & $\begin{array}{c}\alpha \\
(\operatorname{deg} \mathbf{J} 2000.0)\end{array}$ & $\begin{array}{c}\delta \\
(\operatorname{deg} \mathbf{J} 2000.0)\end{array}$ & $\begin{array}{c}g \\
(\mathrm{mag})\end{array}$ & $\begin{array}{c}\delta g \\
(\mathrm{mag})\end{array}$ & $\begin{array}{c}A_{g} \\
(\mathrm{mag})\end{array}$ & $\begin{array}{c}r \\
(\mathrm{mag})\end{array}$ & $\begin{array}{c}\delta r \\
(\mathrm{mag})\end{array}$ & $\begin{array}{c}A_{r} \\
(\mathrm{mag})\end{array}$ \\
\hline 0 & 358.88627 & -59.427008 & 18.20 & 0.01 & 0.040 & 17.43 & 0.01 & 0.03 \\
\hline 1 & 358.89465 & -59.422329 & 19.64 & 0.01 & 0.040 & 19.07 & 0.01 & 0.03 \\
\hline 3 & 358.90786 & -59.681289 & 18.28 & 0.01 & 0.038 & 16.78 & 0.01 & 0.03 \\
\hline 4 & 358.91664 & -59.438449 & 18.69 & 0.01 & 0.040 & 17.29 & 0.01 & 0.03 \\
\hline
\end{tabular}

(This table is available in its entirety in machine-readable form.) 


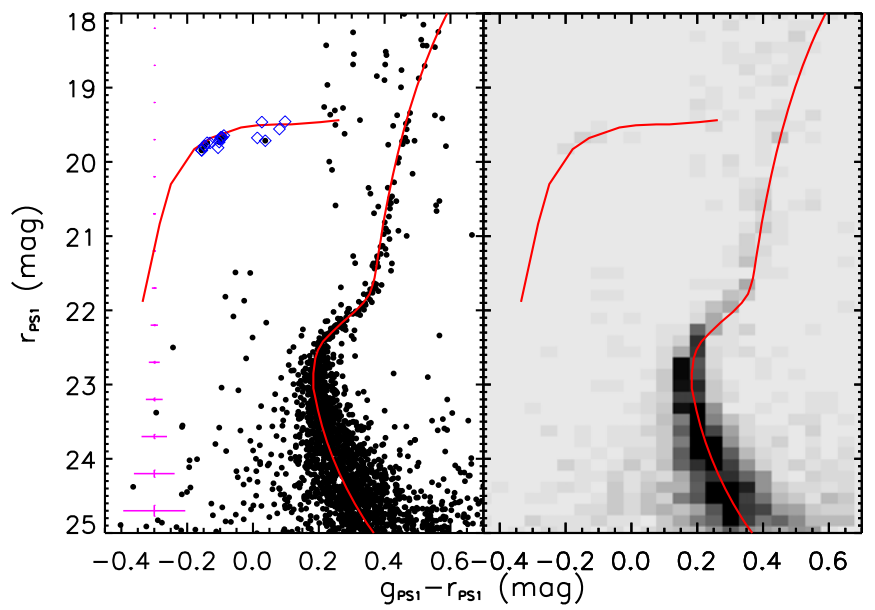

Figure 1. Left: CMD of Sgr II, including stars within one half-light radius of its center. Magenta error bars show the color and magnitude uncertainties as a function of $r$ magnitude. Blue open diamonds are blue $\mathrm{HB}$ candidates within our FoV. There are a total of 19 candidates, 10 of which are within its half-light radius. Right: background-subtracted binned Hess diagram of Sgr II for the same selected region shown in the left panel. Overplotted as a red line is the PS1 fiducial for M15 from Bernard et al. (2014).

the number density of stars in the selected region. The background is estimated from a field located outside a radius of $12^{\prime}$, well outside the body of each satellite. For Ret II and Tuc III, we use background fields that are northwest and southeast of the centroids, because there should be little satellite contamination at these positions, given the position angle and high ellipticity of Ret II (see Table 6) and the orientation of Tuc III stream (see Section 4.4). In Figure 2, overplotted as a red line is a metal-poor Dartmouth isochrone (Dotter et al. 2008), which corresponds to the best-fit Dartmouth isochrone in Section 3.2. In Figure 1, we use the PS1 fiducial for globular cluster NGC 7078 (M15) from Bernard et al. (2014), which is a better fit for Sgr II than any theoretical isochrone. Note that there is not any established DES fiducial for globular clusters, which is why we display theoretical isochrones in Figure 2. We shift each isochrone and fiducial by the best-fit distance modulus that we derive in Section 3.2.

\subsection{Distance}

We derive the distance to Sgr II by comparing its CMD with empirical globular cluster fiducials and theoretical isochrones. We use four empirical fiducials determined by Bernard et al. (2014) using the PS1 photometry NGC 7078 (M15), NGC 6341 (M92), NGC 6205 (M13), and NGC 5272 (M3) with $[\mathrm{Fe} / \mathrm{H}]=-2.42,-2.38,-1.60$, and -1.50 (Kraft \& Ivans 2003). We assume the distance modulus values of $m-M=15.25$ for M15, 14.75 for M92, 14.42 for M13, and 15.02 for M3 (Kraft \& Ivans 2003), with an uncertainty of 0.1 mag. Besides these four empirical fiducials, we also use the Dartmouth isochrones with $[\mathrm{Fe} / \mathrm{H}]=-2.20,[\alpha / \mathrm{Fe}]=0.40$, and a $13.5 \mathrm{Gyr}$ stellar population.

To determine the distance modulus of Sgr II, we follow a very similar methodology as that described in Sand et al. (2009; see also Walsh et al. 2008). We include all stars with $r<24$ mag within $r_{h}=1 ! 58$ of its center. Each fiducial is shifted through $0.025 \mathrm{mag}$ intervals in $(m-M)$ from 18.0 to 20.0 mag. In each step, we count the number of stars consistent with the fiducial, taking into account photometric uncertainties. The selection region is defined by two selection boundaries on either side along the $g-r$ color axis at the typical color uncertainty at a given $r$ magnitude, as determined via our artificial star tests. We also account for background stars by running the identical procedure in parallel over an appropriately scaled background region offset from Sgr II, counting the number of stars consistent with the fiducial and then subtracting this number from that derived at the position of the dwarf. We derive the best-fit distance modulus when the fiducial gives the maximum number of dwarf stars: 19.250 for M15 (150 stars), 19.125 for M92 (170 stars), 18.675 for M13 (93 stars), and 18.700 for M3 (68 stars). Sgr II's CMD is clearly more consistent with the metal-poor fiducials, i.e., M15 and M92. The Dartmouth isochrone gives $(m-M)=19.225$ mag with 112 stars. We use a 100 iteration bootstrap analysis to determine the uncertainties on each fit.

We also derive a distance modulus using the possible blue HBs of Sgr II within our FoV (19 stars). We fit to both of the metal-poor fiducial HB sequences (M15 and M92) by minimizing the sum of the squares of the difference between the data and the fiducial. The best-fit distance modulus from HBs is 19.30 for M15 and 19.26 for M92. We calculate the associated uncertainties via jackknife resampling, which accounts for both the finite number of stars and the possibility of occasional interloper stars. We compute the mean and standard deviation of the derived distance moduli from both methods, i.e., fitting the $\mathrm{HB}$ sequence $(19.30,19.26)$ and counting stars from M15 (19.250), M92 (19.125), and the isochrone (19.225). The mean is adopted as our final distance modulus value, and the standard deviation as our uncertainty. The distance modulus uncertainty of the globular clusters and the uncertainties from both jackknife resampling and our bootstrap analysis are added in quadrature to produce our final quoted uncertainty (see Table 6).

We derive the distance to the other satellites by comparing their CMDs with a grid of isochrones from both Bressan et al. (2012) and Dotter et al. (2008), using the star counting technique described above. We use a $13.5 \mathrm{Gyr}$ stellar population with a range of metallicities $([\mathrm{Fe} / \mathrm{H}]=-2.4,-2.2,-2.0,-1.7,-1.5)$. Similar to Sgr II, the best-fit distance modulus values are found when the distanced-shifted isochrones give the maximum number of dwarf stars with $r<24$ mag. For Phe II, which has very few RGB stars, we extend the limiting magnitude to $r=25$ mag to capture a larger number of main-sequence stars. The best-fit Dartmouth and PARSEC isochrones are the ones with $[\mathrm{Fe} / \mathrm{H}]=-2.4$ and $[\mathrm{Fe} / \mathrm{H}]=-2.0$ for Ret II, $[\mathrm{Fe} / \mathrm{H}]=-2.2$ and $[\mathrm{Fe} / \mathrm{H}]=-2.0$ for Phe II, $[\mathrm{Fe} / \mathrm{H}]=-2.4$ and $[\mathrm{Fe} / \mathrm{H}]=-2.2$ for Tuc III, respectively. We adopt the mean of the results from these isochrones as our final distance moduli, and their standard deviation as our uncertainty. The uncertainties from our bootstrap analysis are added in quadrature to produce our final quoted uncertainty (see Table 6).

\subsection{Structural Properties}

To constrain the structural parameters of our objects, we fit an exponential profile to the $2 \mathrm{D}$ distribution of stars consistent with each satellite by using the maximum likelihood (ML) technique of Martin et al. (2008) as implemented by Sand et al. (2009). In our analysis, we only select stars consistent with the best-fitting Dartmouth isochrone in color-magnitude space after taking into account photometric uncertainties, within our $90 \%$ completeness limit (see Table 1). We inflate the uncertainty to 


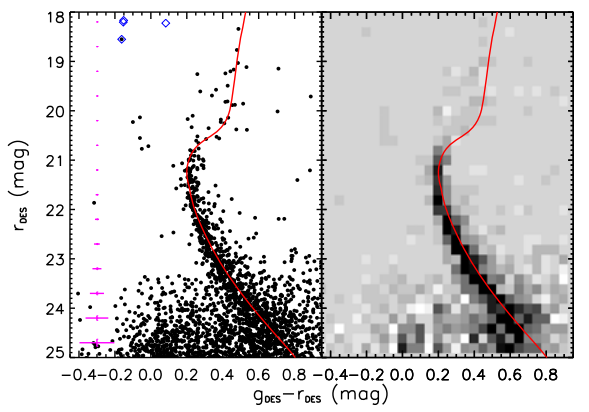

(a) Ret II

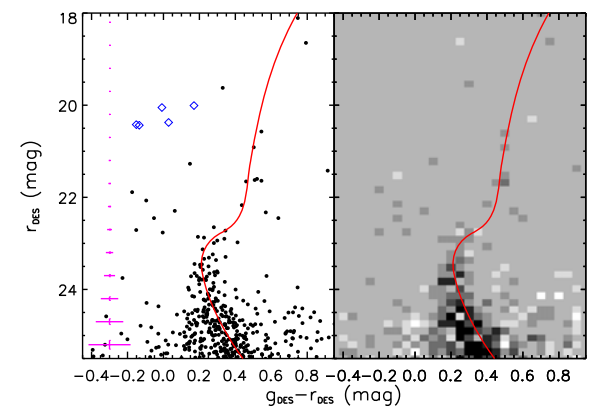

(b) Phe II

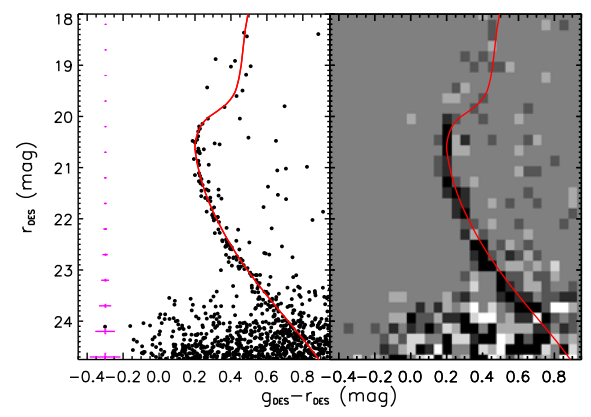

(c) Tuc III

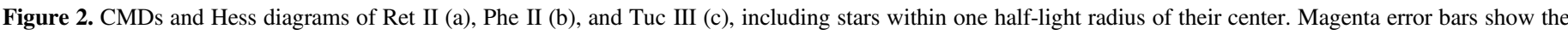

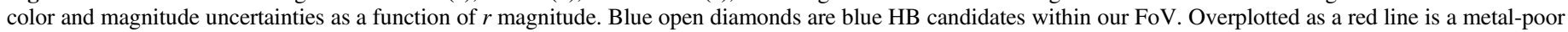
Dartmouth isochrone with age $13.5 \mathrm{Gyr}:[\mathrm{Fe} / \mathrm{H}]=-2.4$ for Ret II and Tuc III, $[\mathrm{Fe} / \mathrm{H}]=-2.2$ for Phe II.

Table 6

Structural Properties

\begin{tabular}{|c|c|c|c|c|}
\hline Parameter & Sgr II & Ret II & Phe II & Tuc III \\
\hline RA (J2000.0) & $19: 52: 39$ ". $53 \pm 3$ !"0 & $03: 35: 47 ! .83 \pm 24 ! .8$ & $23: 39: 58 . ! 27 \pm 8$ !" 3 & $23: 56: 25 ! 80 \pm 36 ! 7$ \\
\hline DEC (J2000.0) & $-22: 03: 54$ ". $19 \pm 2$ ". 2 & $-54: 02: 47 " .80 \pm 9 !^{\prime \prime} 1$ & $-54: 24: 17 ! " 83 \pm 5 ! 7$ & $-59: 34: 59$ ".94 \pm 26 ". 0 \\
\hline$m-M(\mathrm{mag})$ & $19.2 \pm 0.2$ & $17.5 \pm 0.1$ & $19.6 \pm 0.2$ & $16.8 \pm 0.1$ \\
\hline$E(B-V)$ & 0.11 & 0.02 & 0.01 & 0.01 \\
\hline Distance $(\mathrm{kpc})$ & $70.2 \pm 5.0$ & $31.4 \pm 1.4$ & $84.1 \pm 8.0$ & $22.9 \pm 0.9$ \\
\hline$N_{*}^{\mathrm{a}}$ & 1502 & 1120 & 162 & 419 \\
\hline$M_{V}$ & $-5.2 \pm 0.1$ & $-3.1 \pm 0.1$ & $-2.7 \pm 0.4$ & $-1.3 \pm 0.2$ \\
\hline$r_{h}(\operatorname{arcmin})$ & $1.6 \pm 0.1$ & $6.3 \pm 0.4$ & $1.5 \pm 0.3$ & $5.1 \pm 1.2$ \\
\hline$r_{h}(\mathrm{pc})$ & $32 \pm 1$ & $58 \pm 4$ & $37 \pm 6$ & $34 \pm 8$ \\
\hline Ellipticity & $<0.1$ & $0.6 \pm 0.1$ & $0.4 \pm 0.1$ & $0.2 \pm 0.1$ \\
\hline Position angle (deg) & Unconstrained & $68 \pm 2$ & $156 \pm 13$ & $25 \pm 38$ \\
\hline $\log _{10}(J(0.5))\left(\mathrm{GeV}^{2} / \mathrm{cm}^{5}\right)$ & 18.7 & 18.9 & 18.2 & 19.2 \\
\hline$M_{\mathrm{HI}}^{\lim }\left(M_{\odot}\right)$ & 1300 & 430 & 1400 & 210 \\
\hline$M_{\mathrm{H} \text { I }}^{\lim } / L_{V}\left(M_{\odot} / L_{\odot}\right)$ & 0.2 & 0.4 & 2 & 0.5 \\
\hline
\end{tabular}

Note.

${ }^{\mathrm{a}} N_{*}$ is the number of stars brighter than $r=24$ mag selected by the best-fit isochrone for each object.

$0.1 \mathrm{mag}$ when the photometric errors are $<0.1$ mag for the purpose of selecting stars to go into our ML analysis. For Tuc III, a limiting magnitude of $r=24 \mathrm{mag}$ is used to avoid contamination from field stars and unresolved background galaxies. The resulting structural parameters are summarized in Table 6, which includes the central position, half-light radius $\left(r_{h}\right)$, ellipticity $(\epsilon)$, and position angle. The quoted $r_{h}$ is the bestfit elliptical half-light radius along the semimajor axis. Uncertainties are determined by bootstrap resampling the data 1000 times, and recalculating the structural parameters for each resample. We check our results by repeating the calculations with the same set of stars, but with a limit one magnitude brighter. The derived structural parameters using both samples of stars are consistent within the uncertainties.

Recently, Muñoz et al. (2012) presented a suite of simulations of low-luminosity MW satellites under different observing conditions to recover structural parameters within $10 \%$ or better of their true values: they suggested a FoV at least three times that of the half-light radius being measured, greater than 1000 stars in the total sample, and a central density contrast of 20 over the background. These conditions are satisfied in our data for all but one satellite, Tuc III. Our sample of Tuc III has $<500$ stars and a central density contrast of $\sim 15$; moreover, Tuc III is clearly a disrupting system (e.g., DrlicaWagner et al. 2015; Shipp et al. 2018; see also Section 4.4), whose true structural parameters may be difficult to gauge with our ML technique.

In Figure 3, we show 1D stellar radial profiles, along with our best-fit exponential profile derived from the best-fit 2D stellar distribution. We use elliptical bins based on the parameters from the ML analysis. The 1D representations of the exponential fit and the data are in good agreement, but we also note that parameterized models, condensed to $1 \mathrm{D}$, cannot capture a satellite's potentially complex structure. For Tuc III, there are two bright stars that lead to incompleteness in our star counts, as shown with a blue arrow (see also Figure 4). We also note that the radial density profiles of Ret II and Tuc III do not reach the background level, while those of Sgr II and Phe II barely do. This limits our ability to investigate the outer regions of these satellites.

\subsection{Absolute Magnitude}

We derive absolute magnitudes for our objects by using the same procedure as in Sand et al. (2009, 2010), as was first described in Martin et al. (2008). First, we build a wellpopulated CMD (of 20,000 stars), including our completeness and photometric uncertainties, by using the best-fit Dartmouth isochrone (Sgr II and Phe II: $[\mathrm{Fe} / \mathrm{H}]=-2.2$; Ret II and Tuc III: $[\mathrm{Fe} / \mathrm{H}]=-2.4$ ) and its associated luminosity function with a Salpeter initial mass function. Then, we 

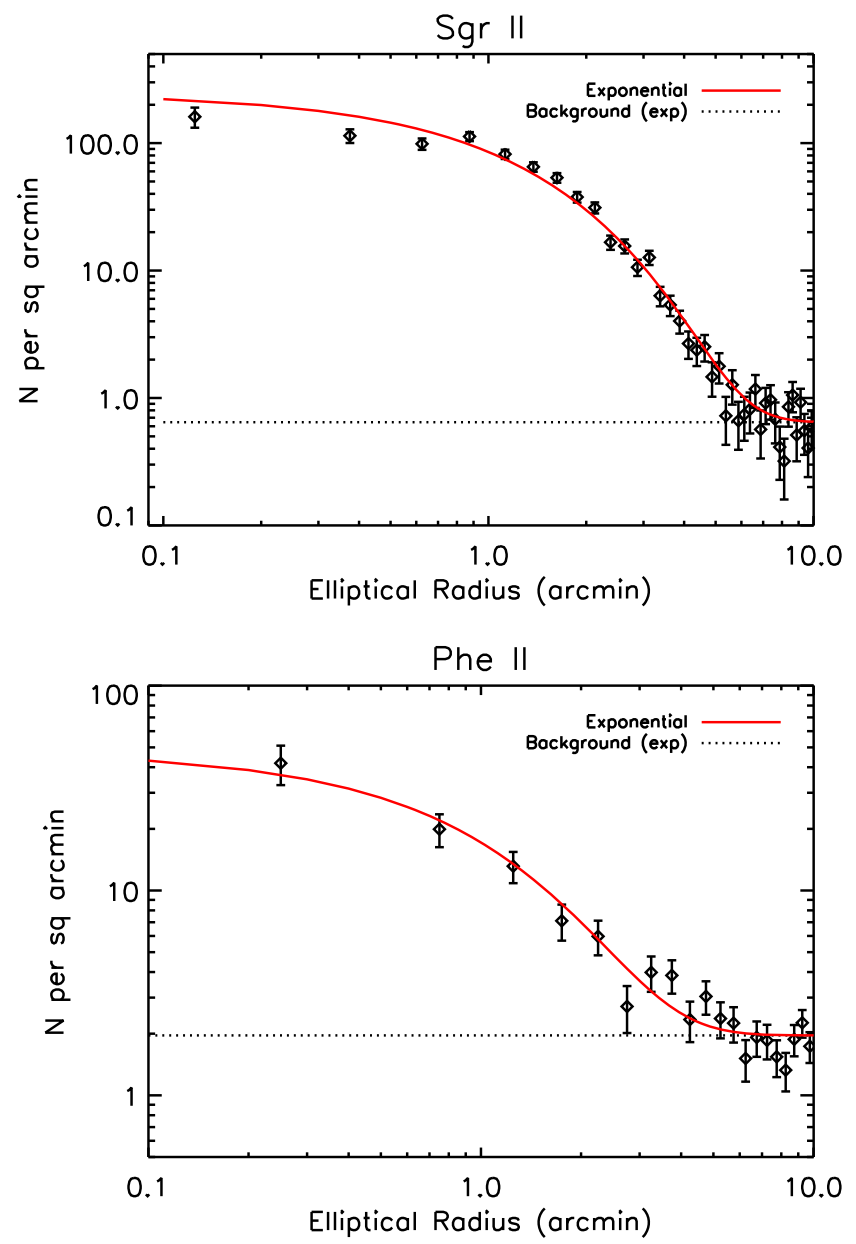

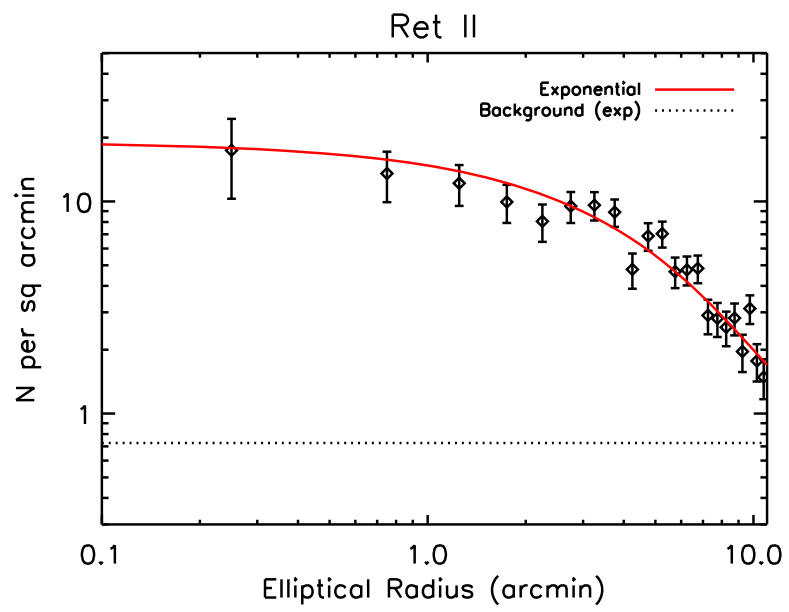

Tuc III

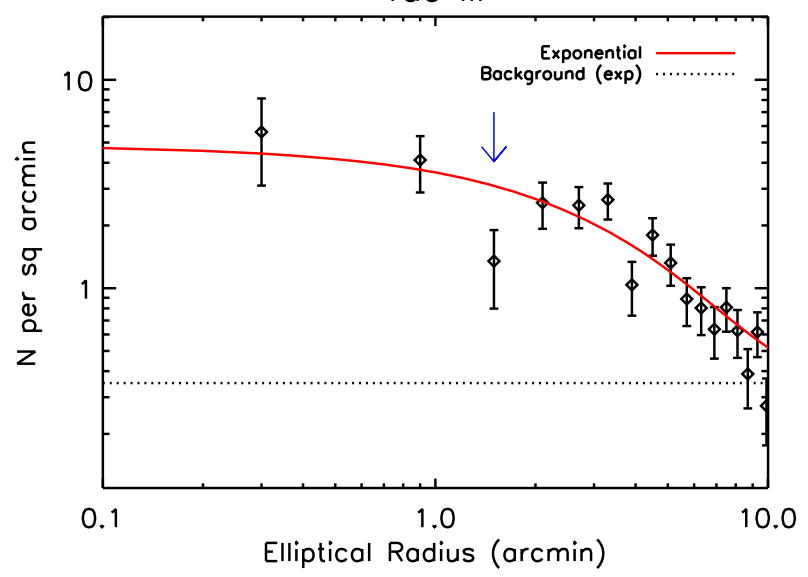

Figure 3. Stellar profiles of Sgr II, Ret II, Phe II, and Tuc III. The red and dotted lines show the best-fit 1D exponential and the background surface density, found via our ML analysis, respectively. In Tuc III, the blue arrow highlights the bin where the presence of two bright stars causes a dip in the profile. Note that our profile fits are derived from the 2D stellar distribution, not from the 1D profile.

randomly select the same number of stars from this artificial CMD as was found from our exponential profile fits (over the same magnitude range as was used for the ML analysis). We correct the number of stars of Tuc III for the bin obscured by bright stars, assuming that this bin (highlighted by blue arrow in Figure 3) has a stellar density that lands on the exponential fit. We sum the flux of these stars, and extrapolate the flux of unaccounted stars using the adopted luminosity function. We calculate 1000 realizations in this way, and take the mean as our absolute magnitude and its standard deviation as our uncertainty. To account for the distance modulus uncertainty and the uncertainty on the number of stars, we repeat this operation 100 times, varying the presumed distance modulus and number of stars within their uncertainties, and use the offset from the best-fit value as the associated uncertainty. All of these error terms are then added in quadrature to produce our final uncertainty on the absolute magnitude. We note that the Dartmouth isochrone accounts for red giant branch (RGB) and main-sequence stars but not HB sequences. Adding the fluxes of our HB candidates gives the total absolute magnitude of $M_{V}=-5.2 \mathrm{mag}$ for Sgr II, $-3.1 \mathrm{mag}$ for Ret II and $-2.7 \mathrm{mag}$ for Phe II, which we adopt as our final values. For each satellite, the effect of HB candidates on the absolute magnitude is within our quoted uncertainty. Note that Tuc III does not have any $\mathrm{HB}$ candidates.

\subsection{Extended Structure Search}

Given photometric hints that some new MW satellites may be tidally disturbed (e.g., Sand et al. 2009; Muñoz et al. 2010, among others), we search for any sign of tidal interaction, such as streams or other extensions, within our data. We use a matched-filter technique similar to Sand et al. (2012), as was originally described in Rockosi et al. (2002). This method maximizes the signal-to-noise in possible satellite stars over the background. As signal CMDs, we use the artificial ones that are created in Section 3.4 to derive the absolute magnitudes. For background CMDs, we use stars from a field well outside the body of each satellite. We bin these CMDs into $0.15 \times 0.15$ color-magnitude bins.

Figure 4 shows our final smoothed matched-filter maps, where we have spatially binned the input data, and smoothed with a Gaussian of width 1.5 times that of the pixel size. Spatial bins for Sgr II and Ret II are 20", while for Phe II and Tuc III we used spatial pixels of $25^{\prime \prime}$. The background of these smoothed maps is determined using the MMM routine in IDL. The main body of each satellite is clearly visible in each map. Magenta diamonds show blue HB candidates if there are any. In the Tuc III map, scaled cyan circles highlight the position of bright stars and the approximate size of their halos; in these regions, our stellar catalogs are compromised, which translates to holes in our spatial maps. 

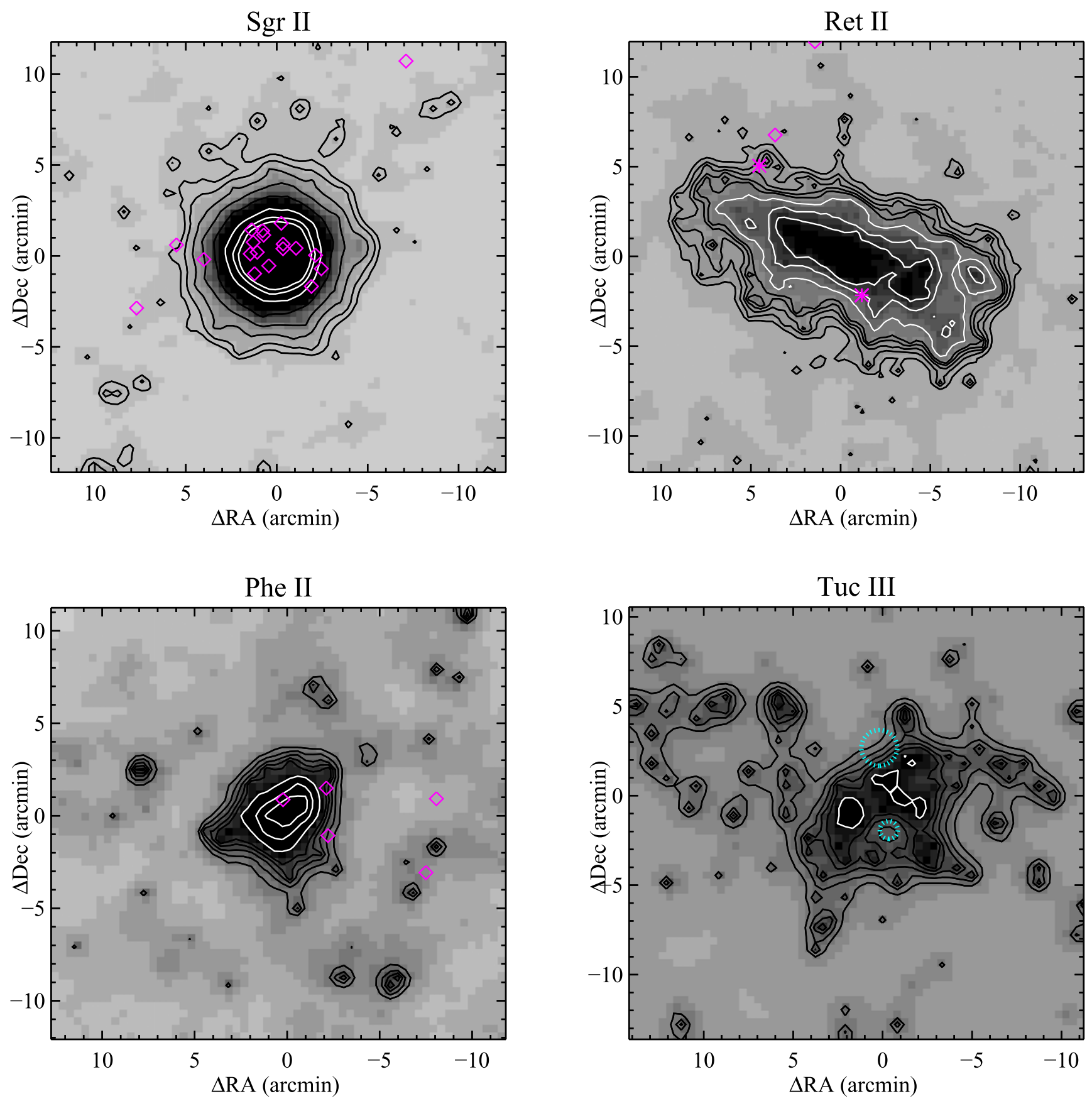

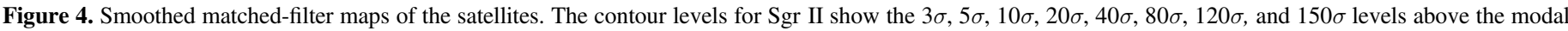

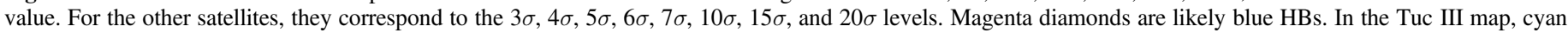
circles highlight the presence of bright stars which cause stellar incompleteness at those positions.

\subsection{Neutral Hydrogen Content}

We constrain the neutral hydrogen (H I) content of our objects by mining the HI Parkes All-Sky Survey (HIPASS; Barnes et al. 2001; Kalberla \& Haud 2015) and Galactic AllSky Survey (GASS; McClure-Griffiths et al. 2009) along the lines of sight given in Table 6. We find no evidence for $\mathrm{HI}$ emission within the half-light radii of the objects at heliocentric velocities in the range $-400 \mathrm{~km} \mathrm{~s}^{-1}<\mathrm{V}_{\text {hel }}<400 \mathrm{~km} \mathrm{~s}^{-1}$, beyond that from the HI layer of the MW itself at $V_{\text {hel }} \sim 0$ $\mathrm{km} \mathrm{s}^{-1}$. We therefore place $5 \sigma$ upper limits on the H I mass $M_{\mathrm{H} \text { I }}^{\lim }$ of each object, using stellar radial velocity measurements to constrain the search when possible. The resulting $M_{\mathrm{HI}}^{\lim }$ and $M_{\mathrm{H} \mathrm{I}}^{\lim } / L_{V}$ are given in Table 6.

The measured stellar radial velocity centroids of Ret II $\left(V_{\mathrm{sys}}=[62.8 \pm 0.5] \mathrm{km} \mathrm{s}^{-1}\right.$, Simon et al. 2015) and Tuc III $\left(V_{\mathrm{sys}}=[-102.3 \pm 0.4 \mathrm{stat} \pm 2.0 \mathrm{sys}] \mathrm{km} \mathrm{s}^{-1}\right.$, Simon et al. 2017) place them within and beyond the range of $V_{\text {hel }}$ contaminated by MW H I emission, respectively, at the GASS sensitivity. The upper limit on the H I mass $M_{\mathrm{HI}}^{\lim }$ of Ret II derived by Westmeier et al. (2015) using HIPASS (before a $V_{\text {sys }}$ for this object was published) is only valid if it is well separated from the MW H I layer in velocity; this is clearly not 


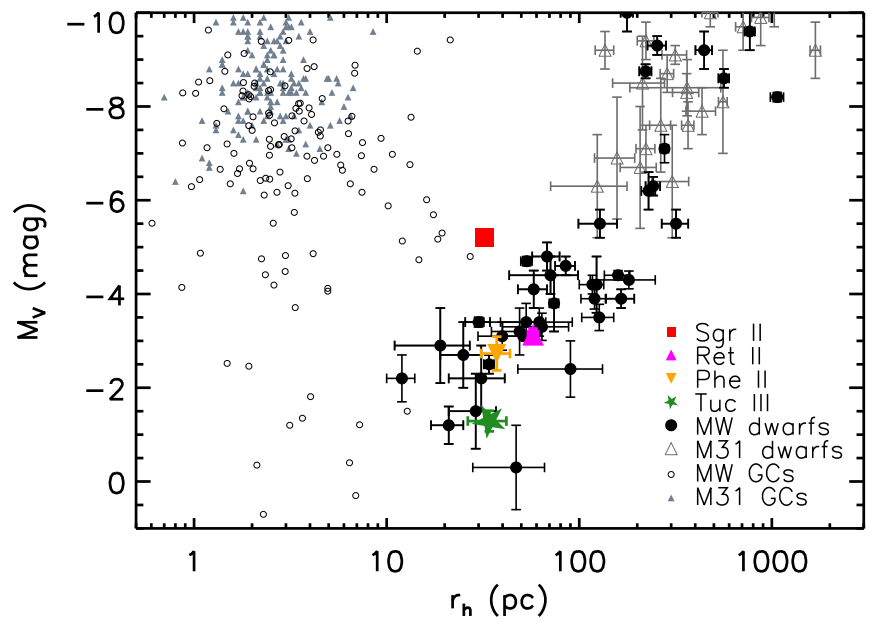

Figure 5. Absolute magnitude $\left(M_{V}\right)$ vs. half-light radius $\left(r_{h}\right)$, showing M31 globular clusters (small filled gray triangles; Strader et al. 2011), M31 satellite galaxies (open gray triangles; McConnachie 2012), MW globular clusters (small open black circles; Belokurov et al. 2010; Harris 2010; Muñoz et al. 2012; Balbinot et al. 2013; Laevens et al. 2014; Kim \& Jerjen 2015; Kim et al. 2015b, 2016), and MW dwarfs (filled black circles; McConnachie 2012; Bechtol et al. 2015; Drlica-Wagner et al. 2015; Kim \& Jerjen 2015; Koposov et al. 2015a, 2018; Laevens et al. 2015; Crnojević et al. 2016; Drlica-Wagner et al. 2016; Homma et al. 2016, 2018; Kim et al. 2016; Torrealba et al. 2016; Carlin et al. 2017). Our objects are displayed with a filled red square (Sgr II), filled magenta triangle (Ret II), filled yellow upside down triangle (Phe II), and filled green star (Tuc III). The size of the error range for Sgr II is less than the symbol size.

the case. We therefore derive a physically meaningful $M_{\mathrm{H} \text { I }}^{\lim }$ using the GASS data (which, unlike HIPASS, accurately recover large-scale $\mathrm{H}$ I emission) at $V_{\text {sys }}$ for Ret II, smoothing to a spectral resolution of $10 \mathrm{~km} \mathrm{~s}^{-1}$ and adopting this velocity width in the upper limit. As Tuc III is well separated from the MW H I layer, we use the more sensitive HIPASS data to derive $M_{\mathrm{H}}^{\lim }$ in a single $26.4 \mathrm{~km} \mathrm{~s}^{-1}$-wide Hanning-smoothed channel.

Stellar radial velocity measurements for Sgr II and Phe II are not available in the literature. For Sgr II, we compute $M_{\mathrm{H} \text { I }}^{\lim }$ from HIPASS as described for Tuc III above; for Phe II, we use the upper limit obtained by Westmeier et al. (2015) from reprocessed HIPASS data, adjusting $M_{\mathrm{HI}}^{\lim }$ to the distance reported in Table 6 . We emphasize that these $M_{\mathrm{H} \text { I }}^{\text {lim }}$ are valid only if $V_{\text {sys }}$ for Sgr II and Phe II are well separated from MW emission along their respective lines of sight. Using the GASS data to identify the velocity ranges contaminated by the MW, we find that $M_{\mathrm{HI}}^{\lim }$ is valid for Sgr II if it has $V_{\text {sys }} \lesssim$ $-100 \mathrm{~km} \mathrm{~s}^{-1}$ or $V_{\text {sys }} \gtrsim 125 \mathrm{~km} \mathrm{~s}^{-1}$, and $M_{\mathrm{HI}}^{\text {lim }}$ is valid for Phe II $V_{\text {sys }} \lesssim-80 \mathrm{~km} \mathrm{~s}^{-1}$ or $V_{\text {sys }} \gtrsim 110 \mathrm{~km} \mathrm{~s}^{-1}$.

The upper limits on the gas richness $M_{\mathrm{H} \text { I }}^{\lim } / L_{V}$ for Sgr II, Ret II, and Tuc III imply that these objects are gas poor, while $M_{\mathrm{H} \text { I }}^{\lim } / L_{V}$ for Phe II does not rule out the possibility of a gas reservoir similar to that of gas-rich galaxies in the Local Volume (Huang et al. 2012; Bradford et al. 2015). The general lack of $\mathrm{HI}$ in these objects is consistent with their location within the virial radius of the MW and M31, within which all low-mass satellites are devoid of gas (e.g., Grcevich \& Putman 2009; Spekkens et al. 2014; Westmeier et al. 2015).

\section{Discussion}

Figure 5 displays the absolute magnitude $\left(M_{V}\right)$ versus halflight radius $\left(r_{h}\right)$ of our objects in the context of M31 and MW satellites. Small filled gray triangles are M31 globular clusters from Strader et al. (2011), and open gray triangles are M31 satellite galaxies from McConnachie (2012). Small open black circles represent MW globular clusters from the Harris (2010) catalog, supplemented by more recent discoveries (Belokurov et al. 2010; Muñoz et al. 2012; Balbinot et al. 2013; Laevens et al. 2014; Kim \& Jerjen 2015; Kim et al. 2015b, 2016). Filled black circles show MW dwarfs from McConnachie (2012), including recently discovered MW dwarf candidates (Bechtol et al. 2015; Drlica-Wagner et al. 2015; Kim \& Jerjen 2015; Koposov et al. 2015a, 2018; Laevens et al. 2015; DrlicaWagner et al. 2016; Homma et al. 2016, 2018; Kim et al. 2016; Torrealba et al. 2016; Carlin et al. 2017). Our objects are displayed with a filled red square (Sgr II), filled magenta triangle (Ret II), filled yellow upside down triangle (Phe II), and filled green star (Tuc III). Sgr II stands out with its intermediate position between the loci of dwarf galaxies and globular clusters in the size-luminosity plane. We discuss each satellite's derived properties in detail in the following subsections.

\subsection{Sgr II}

Our deep photometry provides robust constraints on the structural parameters of Sgr II, and our results are consistent with the discovery analysis of Laevens et al. (2015) within the uncertainties. The CMD of Sgr II (Figure 1) has well-defined features: a narrow RGB and a clear main-sequence turnoff (MSTO), with several blue HB candidates. In Figure 4, magenta diamonds show the spatial position of the HB candidates, most of which are centrally concentrated around Sgr II. Overall, the CMD is consistent with an old stellar population with $[\mathrm{Fe} / \mathrm{H}] \lesssim-2$.

Sgr II occupies an interesting place in the size-luminosity plane (see Figure 5), as it sits in an intermediate position between the loci of dwarf galaxies and globular clusters. Nonetheless, there are several globular clusters with large halflight radii $(>10 \mathrm{pc})$ and an absolute magnitude of $M_{V}>-7$ mag that occupy a similar region in parameter space (see Figure 5). They are mostly distant, and the most metal poor among them (NGC 5053) has $[\mathrm{Fe} / \mathrm{H}]=-2.27$ (Harris 2010), a value that is consistent with the photometric metallicity of Sgr II. Adding to this, Sgr II is very round, with $\epsilon<0.1$, which is consistent with the bulk of MW GCs, although there are definite dwarf galaxies with similar ellipticities (for instance, Leo II; Coleman et al. 2007a).

The ensemble properties of Sgr II (i.e., distance, luminosity, and size) are comparable to that of Palomar 14 (Pal 14), which is one of the most distant $(D=71 \pm 2 \mathrm{kpc})$, faint $\left(M_{V}=\right.$ $-4.9 \pm 0.1 \mathrm{mag})$, and diffuse $\left(r_{h}=46 \pm 3 \mathrm{pc}\right)$ outer Galactic halo globular clusters (Sollima et al. 2011). ${ }^{9}$ Similar to Sgr II, the CMD of Pal 14 presents a narrow RGB (Sollima et al. 2011). However, Sgr II is more metal poor than Pal 14 $([\mathrm{Fe} / \mathrm{H}] \sim-1.5)$. On the basis of its existing tidal tail, Pal 14 was suggested to be a part of a stream consisting of the Fornax dSph and globular cluster Palomar 15 (Sollima et al. 2011).

A population of extended, diffuse star clusters have recently been discovered around M31 (Huxor et al. 2014) with roughly similar properties to Sgr II. Most of these systems are far from M31 itself ( $>30 \mathrm{kpc}$ ), and many appear to be associated with

\footnotetext{
9 Note that in Figure 5 we plot the properties of MW globular clusters from Harris (2010), which report $r_{h}=27 \mathrm{pc}$ and $M_{V}=-4.8$ mag for Pal 14 .
} 
streams (Chapman et al. 2008; Forbes \& Bridges 2010; Mackey et al. 2010). This supports the picture described by Laevens et al. (2015) in which Sgr II was brought into the MW halo along with the Sgr stream, and similar to numerous other MW and M31 GCs (e.g., Law \& Majewski 2010; Mackey et al. 2013).

Despite the strong hints presented above that Sgr II is likely an extended globular cluster, ultimately spectroscopic followup is necessary to determine its true nature.

\subsection{Ret II}

In Figure 2, the CMD of Ret II shows a well-defined main sequence, with several blue $\mathrm{HB}$ candidates that trace its density contours. On the basis of their velocities, two of our candidates (shown with magenta stars in Figure 4) are the confirmed members of Ret II (Simon et al. 2015). We note that other two were not studied by Simon et al. (2015). Our distance measurement $(\mathrm{D}=32 \pm 1 \mathrm{kpc})$ is consistent with both independent discovery analyses (Bechtol et al. 2015; Koposov et al. 2015a). Our ML analysis suggests a similar value for its half-light radius $\left(r_{h}=58 \pm 4 \mathrm{pc}\right)$ to the result of (Bechtol et al. 2015, $r_{h}=55 \pm 5 \mathrm{pc}$ ), and this is also marginally consistent with the result $^{10}$ of Koposov et al. (2015a). Our absolute magnitude measurement $\left(M_{V}=-3.1 \pm 0.1\right)$ is in between the results of (Koposov et al. 2015a, $M_{V}=-2.7 \pm 0.1$ ) and (Bechtol et al. $\left.2015, M_{V}=-3.6 \pm 0.1\right)$. Ret II is among the most elongated of the MW satellites with $\epsilon \sim 0.6$, comparable to Hercules $(\epsilon \sim 0.7)$ and Ursa Major I $(\epsilon \sim 0.8)$, both of which are at $D \gtrsim 100 \mathrm{kpc}$ (see Table 7 of Sand et al. 2012). Therefore, Ret II is the most elongated nearby dwarf galaxy currently known for its luminosity range. In spite of its elongated nature, our density map does not show any clear sign of tidal features within our FoV (see Figure 4). Deep wide-field observations of Ret II are necessary to truly search for signs of extended structure.

Ret II lands directly on the locus defined by MW ultra-faint dwarf galaxies of similar luminosity (see Figure 5); spectroscopic follow-up (Koposov et al. 2015b; Simon et al. 2015; Walker et al. 2015) confirmed that it is a MW ultra-faint dwarf galaxy on the basis of the velocities and metallicities of its stars. They found that Ret II is strongly dark matter-dominated, and one of the most metal-poor galaxies known with a mean metallicity of $[\mathrm{Fe} / \mathrm{H}]<-2.5$. As expected from these studies, an old metal-poor Dartmouth isochrone $([\mathrm{Fe} / \mathrm{H}]=-2.40,[\alpha /$ $\mathrm{Fe}]=0.40,13.5 \mathrm{Gyr}$ ) provides the best fit to the features of its CMD at our measured distance (see Figure 2).

A tentative gamma-ray detection associated with Ret II (at the $\sim 2-3 \sigma$ level; Geringer-Sameth et al. 2015), led to excitement that a signal from annihilating dark matter was found, but this detection is not seen by all of the searching teams (see Drlica-Wagner et al. 2015). Using the photometric analysis of Bechtol et al. (2015); Simon et al. (2015) conclude that Segue 1, Ursa Major II and Coma Berenices, which possess larger J-factors, are more promising gamma-ray targets than Ret II. Using our photometric analysis and the scaling relation of Pace \& Strigari (2018), we find the same value for the J-factor and confirm their conclusion.

\footnotetext{
${ }^{10}$ Koposov et al. (2015a) reported the azimuthally averaged half-light radius of $32 \mathrm{pc}$. Their value after correcting for the ellipticity is $r_{h}=50_{-2}^{+3} \mathrm{pc}$.
}

\subsection{Phe II}

The CMD features of Phe II are much clearer in the Hess diagram, which accounts for the contaminating background stars (see Figure 2). Phe II has a sparsely populated RGB, with a potential population of blue HBs outside our half-light radius (see Figure 4).

The structural parameters of Phe II are not well constrained in the discovery analyses (Bechtol et al. 2015; Koposov et al. 2015a). Our deep photometry provides robust constraints on these parameters, which mostly agree well with the results from Koposov et al. (2015a), within the uncertainties. Compared with their estimations, our ML analysis suggests a similar size $\left(r_{h}=37 \pm 6 \mathrm{pc} \text { versus } 35.7_{-5}^{+8} \mathrm{pc}\right)^{11}$ and a slightly rounder shape $\left(\epsilon=0.4 \pm 0.1\right.$ versus $\left.0.5_{-0.3}^{+0.1}\right)$ with similar luminosity $\left(M_{V}=-2.7 \pm 0.4\right.$ versus $\left.-2.8 \pm 0.2\right)$ for Phe II. We note our absolute magnitude is fainter than that in Bechtol et al. (2015) $\left(M_{V}=-3.7 \pm 0.4\right)$, however their value would be $M_{V}=-3.4 \pm 0.4$ if shifted by our distance modulus ( $m-\mathrm{M}=19.6)$. In Figure 5, it lands directly on the locus defined by other MW ultra-faint dwarf galaxies of similar luminosity. Judging from the ellipticity and position in the size-luminosity plane, it is likely that Phe II is a dwarf galaxy.

\subsection{Tuс III}

Our deep photometry of Tuc III reveals a well-defined narrow main sequence consistent with old, metal-poor stellar populations, as expected from the spectroscopic metallicity measurement $\left([\mathrm{Fe} / \mathrm{H}]=-2.42_{-0.08}^{+0.07}\right.$, Simon et al. 2017). There are no HB candidates within our FoV, both within our Magellan photometric catalog (which saturates at $r \approx 18 \mathrm{mag}$ ) and the DES catalog, which goes to brighter magnitudes (Drlica-Wagner et al. 2015).

Tuc III is known to host a stellar stream extending at least $\pm 2^{\circ}$ from its core (Drlica-Wagner et al. 2015; Shipp et al. 2018). Despite our narrow FoV and the likelihood that it is contaminated with Tuc III stars throughout, there is still evidence for the stream in our stellar density map (see Figure 4). The presence of bright stars in our field (cyan circles) causes some irregularities in the contours of the satellite body near its center. Despite the existence of a stellar stream, Drlica-Wagner et al. (2015) suggest a relatively low ellipticity for Tuc III. Our ML analysis gives an ellipticity of $\epsilon=0.2 \pm 0.1$ with a major-axis position angle of $\sim 25^{\circ}$. Compared with the discovery analysis $\left(r_{h}=44 \pm 6 \mathrm{pc}\right.$, $M_{V}=-2.4 \pm 0.4$ ), our photometry suggests a similar size $\left(r_{h}=34 \pm 8 \mathrm{pc}\right)$ within the uncertainties, but fainter absolute magnitude $\left(M_{V}=-1.3 \pm 0.2\right)$. The stellar stream together with the narrow FoV and presence of bright stars makes it hard to obtain a robust estimate of Tuc III's structural parameters, therefore our results should be used with caution.

Spectroscopic follow-up (Simon et al. 2017) has been unable to conclusively determine Tuc III's dynamical status and dark matter content. Compared with any known dwarf galaxy, they found a smaller metallicity dispersion and likely a smaller velocity dispersion for Tuc III, and tentatively suggested that it is the tidally stripped remnant of a dark matter-dominated dwarf galaxy. Moreover, its location in the size-luminosity plane favors a ultra-faint dwarf galaxy interpretation (see Figure 5), comparable to Segue I $\left(M_{V}=-1.5 \pm 0.8\right.$,

\footnotetext{
11 Koposov et al. (2015a) reported the azimuthally averaged half-light radius of $26 \mathrm{pc}$. This is their value after correcting for the ellipticity.
} 
$\left.r_{h}=29 \pm 8 \mathrm{pc}\right)$ and Triangulum II $\left(M_{V}=-1.2 \pm 0.4, r_{h}=\right.$ $21 \pm 4 \mathrm{pc})$, both of which have been suggested to be tidally stripped (e.g., Niederste-Ostholt et al. 2009; Kirby et al. 2017).

\section{Summary and Conclusions}

We have presented deep Magellan/Megacam photometric follow-up observations of new MW satellites Sgr II, Ret II, Phe II, and Tuc III. Our photometry reaches $\sim 2-3$ mag deeper than the original discovery data for each object, and allows us to revisit the distance, structural properties, and luminosity measurements. An archival analysis allowed us to place H I gas mass limits on each system, which are all devoid of gas as expected for their location within the virial radius of the MW (e.g., Spekkens et al. 2014).

Sgr II stands in an interesting location in the size-luminosity plane, just between the loci of dwarf galaxies and globular clusters. However, the ensemble of its structural parameters is more consistent with a globular cluster classification. In particular, many of its physical properties are comparable to those of Pal 14. Spectroscopic follow-up is necessary to determine its true nature.

Two independent discovery analyses found different values for the structural parameters of Ret II and Phe II. Our deep photometry resolves this inconsistency, and provides robust constraints on these parameters. We find $M_{V}=-3.1 \pm 0.1$ for Ret II and $M_{V}=-2.7 \pm 0.4$ for Phe II, with corresponding half-light radii of $r_{h \text {, RetII }}=58 \pm 4 \mathrm{pc}$ and $r_{h \text {,PheII }}=37 \pm 6 \mathrm{pc}$. Ret II and Phe II therefore land directly on the locus defined by MW ultra-faint dwarf galaxies of similar luminosity. Ret II is the most elongated nearby dwarf galaxy currently known for its luminosity range, and it is more likely that Phe II is a dwarf galaxy than a star cluster.

Tuc III is extremely faint with $M_{V}=-1.3 \pm 0.2$, and compact $\left(r_{h \text {, TucIII }}=34 \pm 8 \mathrm{pc}\right)$. It is apparently made up of an old, metal-poor stellar population, as expected from its measured spectroscopic metallicity of $[\mathrm{Fe} / \mathrm{H}]=-2.42_{-0.08}^{+0.07}$ (Simon et al. 2017). Our photometry suggests that Tuc III is a tidally disrupted dwarf galaxy.

Finally, we search for any clear sign of extended structure for these satellites (see Figure 4). We find no evidence for structural anomalies or tidal disruption in Sgr II and Phe II. Despite its apparent high ellipticity, Ret II also does not show any firm evidence of extra-tidal material outside the satellite. The stellar density map of Tuc III is of particular interest, because it is known to be associated with a stellar stream. Our deep imaging allows us to map the connection between the stellar steam and the body of Tuc III. However, deep wide-field observations, in particular for Ret II and Tuc III, are necessary to definitively investigate the outer regions of these systems.

Research by D.J.S. and B.M. is supported by NSF grants AST1412504 and AST-1517649. We thank Keith Bechtol for helpful discussions. We also wish to thank the anonymous referee whose comments improved the content of this paper. This paper includes data gathered with the $6.5 \mathrm{~m}$ Magellan Telescopes located at Las Campanas Observatory, Chile. This paper uses data products produced by the OIR Telescope Data Center, supported by the Smithsonian Astrophysical Observatory.

This project used public archival data from the PanSTARRS1 Surveys, which have been made possible through contributions of the Institute for Astronomy, the University of
Hawaii, the Pan-STARRS Project Office, the Max-Planck Society and its participating institutes, the Max Planck Institute for Astronomy, Heidelberg and the Max Planck Institute for Extraterrestrial Physics, Garching, The Johns Hopkins University, Durham University, the University of Edinburgh, Queen's University Belfast, the Harvard-Smithsonian Center for Astrophysics, the Las Cumbres Observatory Global Telescope Network Incorporated, the National Central University of Taiwan, the Space Telescope Science Institute, the National Aeronautics and Space Administration under grant No. NNX08AR22G issued through the Planetary Science Division of the NASA Science Mission Directorate, the National Science Foundation under Grant AST-1238877, the University of Maryland, Eotvos Lorand University (ELTE), and the Los Alamos National Laboratory.

This project used public archival data from the Dark Energy Survey (DES). Funding for the DES Projects has been provided by the U.S. Department of Energy, the U.S. National Science Foundation, the Ministry of Science and Education of Spain, the Science and Technology Facilities Council of the United Kingdom, the Higher Education Funding Council for England, the National Center for Supercomputing Applications at the University of Illinois at Urbana-Champaign, the Kavli Institute of Cosmological Physics at the University of Chicago, the Center for Cosmology and Astro-Particle Physics at the Ohio State University, the Mitchell Institute for Fundamental Physics and Astronomy at Texas A\&M University, Financiadora de Estudos e Projetos, Fundação Carlos Chagas Filho de Amparo à Pesquisa do Estado do Rio de Janeiro, Conselho Nacional de Desenvolvimento Científico e Tecnológico and the Ministério da Ciência, Tecnologia e Inovação, the Deutsche Forschungsgemeinschaft and the Collaborating Institutions in the Dark Energy Survey. The Collaborating Institutions are Argonne National Laboratory, the University of California at Santa Cruz, the University of Cambridge, Centro de Investigaciones Enérgeticas, Medioambientales y Tecnológicas-Madrid, the University of Chicago, University College London, the DESBrazil Consortium, the University of Edinburgh, the Eidgenössische Technische Hochschule (ETH) Zürich, Fermi National Accelerator Laboratory, the University of Illinois at UrbanaChampaign, the Institut de Ciències de lEspai (IEEC/CSIC), the Institut de Física d'Altes Energies, Lawrence Berkeley National Laboratory, the Ludwig-Maximilians Universität München and the associated Excellence Cluster Universe, the University of Michigan, the National Optical Astronomy Observatory, the University of Nottingham, The Ohio State University, the OzDES Membership Consortium, the University of Pennsylvania, the University of Portsmouth, SLAC National Accelerator Laboratory, Stanford University, the University of Sussex, and Texas A\&M University. Based in part on observations at Cerro Tololo Inter-American Observatory, National Optical Astronomy Observatory, which is operated by the Association of Universities for Research in Astronomy (AURA) under a cooperative agreement with the National Science Foundation.

Facility: Las Campanas Observatory: Magellan Clay Telescope/Megacam.

Software: SExtractor (Bertin \& Arnouts 1996), IDL astronomy users library (Landsman 1993), NumPy (Van Der Walt et al. 2011). 


\section{ORCID iDs}

BurÇin Mutlu-Pakdil (i) https://orcid.org/0000-00019649-4815

David J. Sand (1) https://orcid.org/0000-0003-4102-380X

Jeffrey L. Carlin (iD https://orcid.org/0000-0002-3936-9628

Kristine Spekkens (i) https://orcid.org/0000-0002-0956-7949

Nelson Caldwell (iD https://orcid.org/0000-0003-2352-3202

Denija Crnojević (iD https://orcid.org/0000-0002-1763-4128

Beth Willman (iD https://orcid.org/0000-0003-2892-9906

Dennis Zaritsky (i) https://orcid.org/0000-0002-5177-727X

\section{References}

Adén, D., Wilkinson, M. I., Read, J. I., et al. 2009, ApJL, 706, L150

Balbinot, E., Santiago, B. X., da Costa, L., et al. 2013, ApJ, 767, 101

Baltz, E. A., Berenji, B., Bertone, G., et al. 2008, JCAP, 7, 013

Barnes, D. G., Staveley-Smith, L., de Blok, W. J. G., et al. 2001, MNRAS, 322, 486

Bechtol, K., Drlica-Wagner, A., Balbinot, E., et al. 2015, ApJ, 807, 50

Belokurov, V., Irwin, M. J., Koposov, S. E., et al. 2014, MNRAS, 441, 2124

Belokurov, V., Walker, M. G., Evans, N. W., et al. 2010, ApJL, 712, L103

Bergström, L., \& Snellman, H. 1988, PhRvD, 37, 3737

Bernard, E. J., Ferguson, A. M. N., Schlafly, E. F., et al. 2014, MNRAS, 442, 2999

Bertin, E., \& Arnouts, S. 1996, A\&AS, 117, 393

Bradford, J. D., Geha, M. C., \& Blanton, M. R. 2015, ApJ, 809, 146

Bressan, A., Marigo, P., Girardi, L., et al. 2012, MNRAS, 427, 127

Carlin, J. L., Sand, D. J., Muñoz, R. R., et al. 2017, AJ, 154, 267

Chambers, K. C., Magnier, E. A., Metcalfe, N., et al. 2016, arXiv:1612.05560

Chapman, S. C., Ibata, R., Irwin, M., et al. 2008, MNRAS, 390, 1437

Coleman, M. G., Jordi, K., Rix, H.-W., Grebel, E. K., \& Koch, A. 2007a, AJ, 134, 1938

Coleman, M. G., de Jong, J. T. A., Martin, N. F., et al. 2007b, ApJL, 668, L43

Collins, M. L. M., Tollerud, E. J., Sand, D. J., et al. 2017, MNRAS, 467, 573

Conn, B. C., Jerjen, H., Kim, D., \& Schirmer, M. 2018a, ApJ, 852, 68

Conn, B. C., Jerjen, H., Kim, D., \& Schirmer, M. 2018b, ApJ, 857, 70

Crnojević, D., Sand, D. J., Zaritsky, D., et al. 2016, ApJL, 824, L14

DES Collaboration 2018, arXiv:1801.03181

Dotter, A., Chaboyer, B., Jevremović, D., et al. 2008, ApJS, 178, 89

Drlica-Wagner, A., Bechtol, K., Rykoff, E. S., et al. 2015, ApJ, 813, 109

Drlica-Wagner, A., Bechtol, K., Allam, S., et al. 2016, ApJL, 833, L5

Fitzpatrick, E. L. 1999, PASP, 111, 63

Flewelling, H. A., Magnier, E. A., Chambers, K. C., et al. 2016, arXiv:1612. 05243

Forbes, D. A., \& Bridges, T. 2010, MNRAS, 404, 1203

Garling, C., Willman, B., Sand, D. J., et al. 2018, ApJ, 852, 44

Geringer-Sameth, A., Walker, M. G., Koushiappas, S. M., et al. 2015, PhRvL, 115,081101

Grcevich, J., \& Putman, M. E. 2009, ApJ, 696, 385

Gunn, J. E., Lee, B. W., Lerche, I., Schramm, D. N., \& Steigman, G. 1978, ApJ, 223, 1015

Harris, W. E. 2010, arXiv:1012.3224

Homma, D., Chiba, M., Okamoto, S., et al. 2016, ApJ, 832, 21

Homma, D., Chiba, M., Okamoto, S., et al. 2018, PASJ, 70, S18

Hooper, D., \& Linden, T. 2015, JCAP, 9, 016

Huang, S., Haynes, M. P., Giovanelli, R., \& Brinchmann, J. 2012, ApJ, 756, 113

Huxor, A. P., Mackey, A. D., Ferguson, A. M. N., et al. 2014, MNRAS, 442,2165

Kalberla, P. M. W., \& Haud, U. 2015, A\&A, 578, A78
Kim, D., \& Jerjen, H. 2015, ApJ, 799, 73

Kim, D., Jerjen, H., Mackey, D., Da Costa, G. S., \& Milone, A. P. 2015a, ApJL, 804, L44

Kim, D., Jerjen, H., Mackey, D., Da Costa, G. S., \& Milone, A. P. 2016, ApJ, 820,119

Kim, D., Jerjen, H., Milone, A. P., Mackey, D., \& Da Costa, G. S. 2015b, ApJ, 803, 63

Kirby, E. N., Cohen, J. G., Simon, J. D., et al. 2017, ApJ, 838, 83

Koposov, S. E., Belokurov, V., \& Torrealba, G. 2017, MNRAS, 470, 2702

Koposov, S. E., Belokurov, V., Torrealba, G., \& Evans, N. W. 2015a, ApJ, 805,130

Koposov, S. E., Casey, A. R., Belokurov, V., et al. 2015b, ApJ, 811, 62

Koposov, S. E., Walker, M. G., Belokurov, V., et al. 2018, MNRAS, 479, 5343

Kraft, R. P., \& Ivans, I. I. 2003, PASP, 115, 143

Laevens, B. P. M., Martin, N. F., Sesar, B., et al. 2014, ApJL, 786, L3

Laevens, B. P. M., Martin, N. F., Bernard, E. J., et al. 2015, ApJ, 813, 44

Landsman, W. B. 1993, in ASP Conf. Ser. 52, Astronomical Data Analysis Software and Systems II, ed. R. J. Hanisch, R. J. V. Brissenden, \& J. Barnes (San Francisco, CA: ASP), 246

Law, D. R., \& Majewski, S. R. 2010, ApJ, 718, 1128

Mackey, A. D., Huxor, A. P., Ferguson, A. M. N., et al. 2010, ApJL, 717, L11

Mackey, A. D., Huxor, A. P., Ferguson, A. M. N., et al. 2013, MNRAS, 429, 281

Magnier, E. A., Schlafly, E. F., Finkbeiner, D. P., et al. 2016, arXiv:1612.05242

Martin, N. F., de Jong, J. T. A., \& Rix, H.-W. 2008, ApJ, 684, 1075

Martin, N. F., Nidever, D. L., Besla, G., et al. 2015, ApJL, 804, L5

McClure-Griffiths, N. M., Pisano, D. J., Calabretta, M. R., et al. 2009, ApJS, 181,398

McConnachie, A. W. 2012, AJ, 144, 4

McLeod, B., Geary, J., Conroy, M., et al. 2015, PASP, 127, 366

McLeod, B., Geary, J., Ordway, M., et al. 2006, in Scientific Detectors for Astronomy 2005, ed. J. E. Beletic, J. W. Beletic, \& P. Amico (Berlin: Springer), 337

Muñoz, R. R., Geha, M., Côté, P., et al. 2012, ApJL, 753, L15

Muñoz, R. R., Geha, M., \& Willman, B. 2010, AJ, 140, 138

Niederste-Ostholt, M., Belokurov, V., Evans, N. W., et al. 2009, MNRAS, 398, 1771

Pace, A. B., \& Strigari, L. E. 2018, arXiv:1802.06811

Rockosi, C. M., Odenkirchen, M., Grebel, E. K., et al. 2002, AJ, 124, 349

Roderick, T. A., Jerjen, H., Mackey, A. D., \& Da Costa, G. S. 2015, ApJ, 804, 134

Sand, D. J., Olszewski, E. W., Willman, B., et al. 2009, ApJ, 704, 898

Sand, D. J., Seth, A., Olszewski, E. W., et al. 2010, ApJ, 718, 530

Sand, D. J., Strader, J., Willman, B., et al. 2012, ApJ, 756, 79

Schlafly, E. F., \& Finkbeiner, D. P. 2011, ApJ, 737, 103

Schlegel, D. J., Finkbeiner, D. P., \& Davis, M. 1998, ApJ, 500, 525

Shipp, N., Drlica-Wagner, A., Balbinot, E., et al. 2018, arXiv:1801.03097

Simon, J. D., Drlica-Wagner, A., Li, T. S., et al. 2015, ApJ, 808, 95

Simon, J. D., Li, T. S., Drlica-Wagner, A., et al. 2017, ApJ, 838, 11

Skrutskie, M. F., Cutri, R. M., Stiening, R., et al. 2006, AJ, 131, 1163

Sollima, A., Martínez-Delgado, D., Valls-Gabaud, D., \& Peñarrubia, J. 2011, ApJ, 726, 47

Spekkens, K., Urbancic, N., Mason, B. S., Willman, B., \& Aguirre, J. E. 2014, ApJL, 795, L5

Stetson, P. B. 1994, PASP, 106, 250

Strader, J., Caldwell, N., \& Seth, A. C. 2011, AJ, 142, 8

Torrealba, G., Koposov, S. E., Belokurov, V., et al. 2016, MNRAS, 463, 712

Torrealba, G., Belokurov, V., Koposov, S. E., et al. 2018, MNRAS, 475, 5085

Van Der Walt, S., Colbert, S. C., \& Varoquaux, G. 2011, CSE, 13, 22

Walker, M. G., Mateo, M., Olszewski, E. W., et al. 2015, ApJ, 808, 108

Walsh, S. M., Willman, B., Sand, D., et al. 2008, ApJ, 688, 245

Westmeier, T., Staveley-Smith, L., Calabretta, M., et al. 2015, MNRAS, 453,338 